\title{
La activación de la ciudadanía como estrategia para la sostenibilidad turística en lugares patrimoniales cercanos a la gran ciudad. El caso de la región madrileña
}

\author{
Juan Ángel Martín* Marta Domínguez Pérez** \\ Alicia Castillo Mena***: \\ Universidad Complutense de Madrid (España)
}

\begin{abstract}
Resumen: Aunque el Patrimonio Cultural es fuente reconocida de bienestar, su sobreexplotación turística genera fuertes impactos negativos, agravados en las grandes ciudades por la concentración de efectos en el espacio. Este trabajo aborda el caso de Madrid, ciudad que cuenta en su periferia metropolitana con activos patrimoniales de primer nivel, cuyo mejor aprovechamiento turístico ayudaría a mitigar el impacto negativo en la capital, a la vez que contribuiría al desarrollo económico y social de la periferia. En la investigación se aplicó un planteamiento metodológico dinámico con cuatro planos de análisis complementarios: documental y de redes, cualitativo, cuantitativo y procesos participativos. Los resultados evidencian la necesidad de adoptar estrategias de gestión cultural y de planificación turística orientadas al reequilibrio de los flujos de visitantes. La activación de los propios vecinos se revela como un recurso especialmente valioso para fortalecer un turismo de proximidad, experiencial y más sostenible. El impacto de la Covid-19, por una parte, y la declaración del Paseo del Prado y el Retiro como Patrimonio de la Humanidad, por otra, han puesto aún más de actualidad esta situación.
\end{abstract}

Palabras Clave: Turismo cultural; Patrimonio Cultural; Ciudadanía; Turistificación; Sostenibilidad.

The activation of citizenship as a strategy for tourism sustainability in heritage sites near the big city. The case of Madrid region.

Abstract: Although Cultural Heritage is a recognized source of well-being, its touristic overexploitation generates strong negative impacts, aggravated in large cities due to the concentration of effects in space. This work addresses the case of Madrid, a city that has first-rate heritage assets in its metropolitan periphery, whose better touristic use would help to mitigate the negative impact on the capital, while contributing to the economic and social development of the periphery. In the research, a dynamic methodological approach was applied with four complementary analysis planes: documentary and networks, qualitative, quantitative and participatory processes. The results show the need to adopt cultural management and tourism planning strategies aimed at rebalancing visitor flows. The activation of the neighbors themselves is revealed as an especially valuable resource to strengthen proximity, experiential and more sustainable tourism. The The impact of Covid-19, on the one hand, and the declaration of Paseo del Prado and El Retiro as World Heritage Sites, on the other, have made this situation even more topical.

Keywords: Cultural tourism; Cultural Heritage Management; Citizenship; Touristification; Sustainability.

\section{Introducción ${ }^{1}$}

En la actualidad, la turistificación en las grandes ciudades es uno de los temas estrella en el panorama urbano, sobre todo por sus efectos negativos sobre la población local, la pérdida de identidad, la saturación

\footnotetext{
* Universidad Complutense de Madrid (España); E-mail: umartin@ucm.es; https://orcid.org/0000-0003-4766-4552

** Universidad Complutense de Madrid (España); E-mail: madoming@ucm.es; https://orcid.org/0000-0002-3145-6671

**** Universidad Complutense de Madrid (España); E-mail: alicia.castillo@ghis.ucm.es; https://orcid.org/0000-0002-9168-1803
} 
turística, el encarecimiento de la vivienda, etc. (UNWTO, 2013). Siguiendo las directrices de ONU, la sostenibilidad del turismo urbano es uno de los retos que se proponen a las ciudades a través del marco de la Agenda Urbana 2030. Algunas de las principales recomendaciones apuntan a una corrección de la saturación turística (Perkumiene et al, 2019) y, por tanto, a una más equilibrada distribución del turismo en el territorio. Esto es, potenciar la difusión y el esponjamiento que permitan la minimización de los impactos negativos del turismo más intensivo, en aras de la sostenibilidad urbana y la mejora de la calidad de vida. De este modo, tras décadas de énfasis en los aspectos positivos del turismo, a partir de los años noventa del pasado siglo, se plantean discursos críticos centrados en los problemas de tipo social, cultural, etc. (Barrado e Hidalgo, 2019).

También el Patrimonio Cultural tiene una presencia significativa en la Agenda Urbana 2030, que señala su relevancia para el desarrollo local, la cohesión social y el sentimiento de pertenencia, por más que, como se ha señalado por algunos (Gómez, Crespi y Domínguez, 2020), no lo hace de manera explícita ni ampliamente desarrollada. La potencialidad del Patrimonio Cultural más allá de su atractivo turístico se viene demostrando en la ya amplia tradición académica española (Herrero et al, 2006; López Guzmán y Sánchez, 2009; Manero, 2011; Martos, 2012; Rengifo et al, 2015; Andrés, 2015; entre otros), aunque también se ha constatado que los recursos patrimoniales podrán tener un mayor aprovechamiento (VanBlarcom \& Kayahan, 2011; Snowball, 2013; Castillo, 2015; Pérez, 2015; Cavailles et al, 2016; Cortijo \& Pulido, 2016; OMT, 2017; Pérez \& Lois, 2018). Puede afirmarse, por tanto, que si bien el Patrimonio Cultural es un elemento clave de desarrollo local, su sobreexplotación de la mano de un turismo excesivo tiene en algunos casos importantes repercusiones sobre el espacio.

En este trabajo nos acercamos a un escenario que supone un ejemplo ilustrativo de ese complejo entramado y de las dificultades de su articulación. Una gran ciudad, Madrid, que ha visto cómo el incremento de la presión turística produce la saturación de su centro urbano, al tiempo que cuenta con algunos núcleos en su área regional de influencia de gran atractivo por su valor patrimonial y cuyo mayor aprovechamiento turístico podría favorecer, por una parte, el reequilibrio de la actividad turística regional y, por otra, la sostenibilidad urbana de la capital. En tal sentido, la puesta en valor de la ciudadanía ofrece nuevos activos de gran utilidad para potenciar ese reequilibrio regional, al tiempo que se fomenta la identidad local.

La ciudad de Madrid posee un amplio atractivo turístico. Es un destino destacado en los rankings internacionales ${ }^{2}$ que recibe un gran número de turistas ${ }^{3}$, situándose en el primer puesto de entre las ciudades españolas ${ }^{4}$ ya desde hace varias décadas. Sin embargo, también sus impactos negativos son conocidos (Liu, 2003; Saarinen, 2006; Weaver, 2009; García, De la Calle y Mínguez, 2015; Barrado e Hidalgo, 2019). Más allá de sus recursos patrimoniales, Madrid atrae por muchos otros aspectos: cultura, ocio, imagen, compras, negocios, multiculturalidad, etc., lo cual promueve una gran oferta turística, pero que también conlleva la saturación de sus espacios centrales (Barrado e Hidalgo, 2019; Calle, Ferreiro y Mendoza, 2018; Crespi y Domínguez Pérez, 2016).

Sin embargo, en el radio de acción de la ciudad de Madrid, en su región metropolitana, existen otros enclaves de amplio atractivo que podrían jugar un relevante papel como espacios de esponjamiento del turismo. La potenciación de las visitas a dichos espacios, más allá de desplazamientos cortos que además incrementan la movilidad regional (Calle, García y Mínguez, 2015), serviría como mecanismo de redistribución del turismo que gravita sobre Madrid. La reciente incorporación del eje Prado-Retiro al listado de lugares Patrimonio Mundial, lejos de seguir incidiendo en la centralidad de la capital como atractor cultural, podría contribuir, gracias a las sinergias existentes, a esa redistribución de flujos e impactos hacia los tres lugares que han sido objeto de estudio en nuestro trabajo: "Universidad y Barrio Histórico de Alcalá de Henares" (1998), "Paisaje Cultural de Aranjuez" (2001) y "Monasterio y sitio de El Escorial, Madrid" (1984). Declarados Patrimonio Mundial por UNESCO, son exponentes de un gran atractivo turístico y cultural en un entorno regional cuyo centro neurálgico es Madrid capital (Figura 1).

Desafortunadamente, la capacidad de atracción de esos lugares se ve limitada también por la falta de conocimiento específico, afectando en última instancia de manera negativa a la gestión del sector y al reajuste de las estrategias de desarrollo locales y regionales. Existen, no obstante, precedentes destacados que se han ocupado de la transformación de las ciudades patrimoniales y los reales sitios de la Comunidad de Madrid, atendiendo sobre todo a la convivencia entre el Patrimonio, los visitantes y sus habitantes (Troitiño, 2002; Mínguez, 2007; Troitiño, 2012). Nuestro trabajo quiere contribuir a la generación de conocimiento, con un enfoque centrado en el interés por un turismo sostenible (Stabler, 1997) en áreas de influencia amplias, como la región metropolitana, en aras de una descentralización 
Figura 1: Localización en el territorio y áreas respectivas de influencia
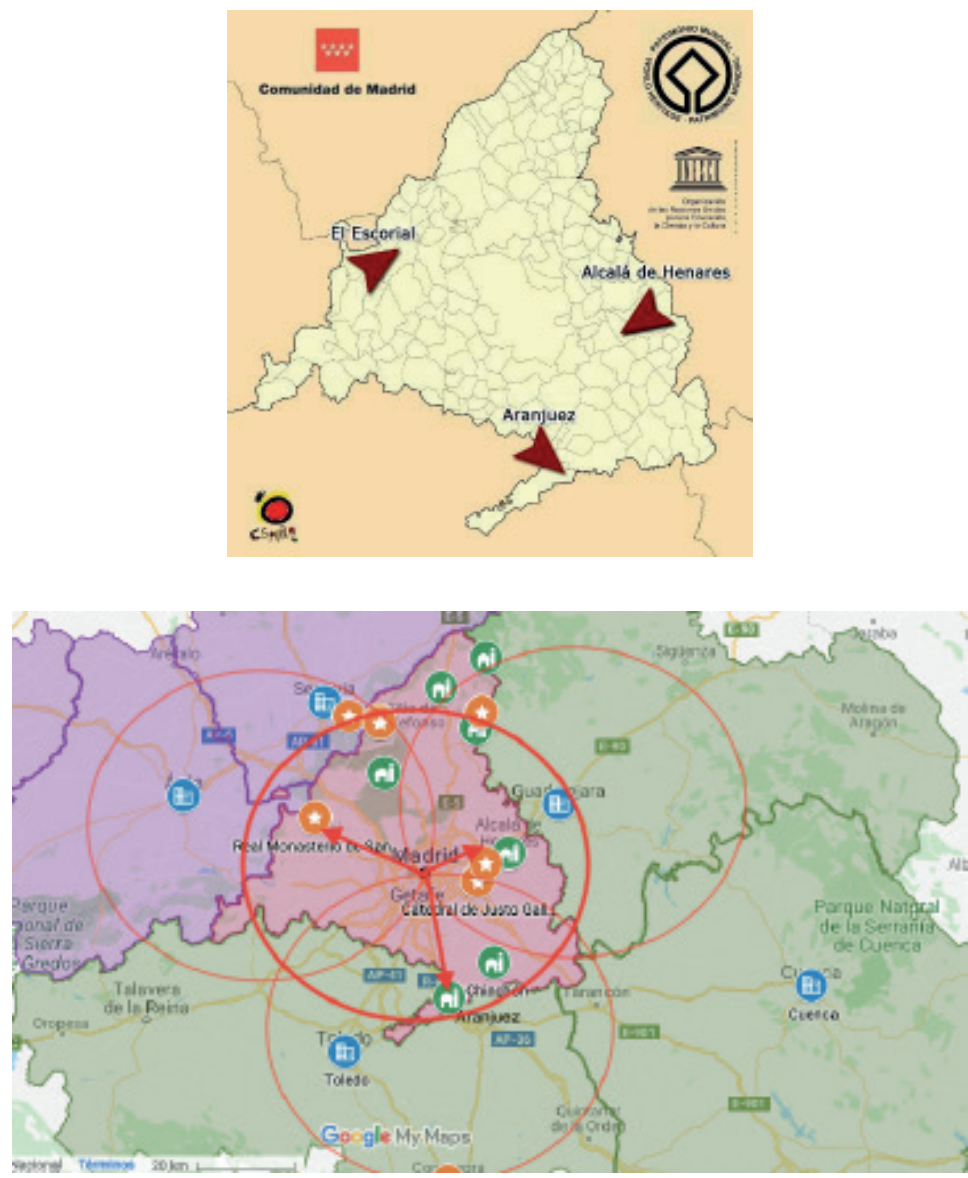

Fuente: Ciudades patrimonio. https://susanaclavero.wordpress.com/2016/05/09/ciudades-patrimonio-mundial-de-la-humanidad-en-la-comunidad-de-madrid/ consultado 8 de julio de 2020.

y diversificación del mismo que, sin dejar de ser un instrumento de desarrollo local, alivie los impactos negativos sobre el centro de las ciudades (Frey y Steiner, 2011; Bonet, 2013; García, De la Calle y Yubero, 2017; Barrado e Hidalgo, 2019). Este tipo de planteamientos se han vuelto incluso más importantes en contextos como el actual, marcado a nivel global por la COVID-19, que está obligando al replanteamiento de algunas estrategias turísticas tradicionales (Arold, 2021) para, en su lugar, potenciar un turismo de proximidad y al margen de los espacios más habituales, desde los centros masificados hacia las periferias en un planteamiento más sostenible.

Consiguientemente, tres áreas constituyen el foco de interés sobre el que se centra el artículo: la estrategia regional de sostenibilidad, la potencialidad multidimensional del Patrimonio Cultural a diferentes escalas -regional y local- y la participación de las poblaciones locales en la gestión del turismo y el patrimonio en las ciudades.

Los resultados obtenidos entendemos que son de utilidad para la articulación de estrategias regionales patrimoniales y turísticas tendentes a reequilibrar los impactos positivos (desarrollo local) y negativos (saturación de los centros urbanos) asociados a estos fenómenos. Las conclusiones alcanzadas no sólo son válidas para el caso concreto aquí analizado, sino también para muchos otros con un escenario similar al que describimos: la presencia de lugares con riqueza patrimonial cercanos a grandes ciudades, las cuales funcionan como nodos de alta atracción turística o que son en sí mismas potentes mercados, lo que da lugar a interesantes sinergias y puede ser utilizado para mejorar la sostenibilidad de los flujos, 
desde entornos saturados hacia otros con potencialidad de acogida. Estas apreciaciones se vuelven aún más significativas tras la incorporación del cuarto espacio madrileño al listado de UNESCO, de cara a articular los flujos de este complejo escenario de forma equilibrada y sostenible. El recurso a la participación de la ciudadanía es una herramienta de incomparable valor para potenciarlos y, en tal sentido, ha resultado muy alentador comprobar cómo los propios vecinos de los municipios estudiados constituyen un recurso de primer orden para articular una mejor relación turista-residente, enriquecer la experiencia de la visita y, al mismo tiempo, fomentar la activación de la ciudadanía.

\section{Fundamentacion teórica}

El Patrimonio Cultural presenta una vertiente de rendimiento multidimensional. Por un lado, una dimensión económica de desarrollo local basada fundamentalmente en el aprovechamiento turístico (Lara de Vicente y López Guzmán, 2004; ICOMOS, 2011) y, por otro, una dimensión más sociológica, cultural y política, ligada a la participación ciudadana y la identificación con un pasado histórico e identitario que revierte en la cohesión social y la gobernanza urbana (Agenda Urbana Española, 2019; Domínguez Pérez, 2019; Gómez, Crespi y Domínguez, 2020).

De este modo, el Patrimonio Cultural juega un papel fundamental en el atractivo turístico y urbano de las ciudades (Zukin, 1995), dando lugar a una demanda "cultural" fundamentada en unos bienes que han de ser considerados como un stock de activos (Throsby, 1998). Estos activos culturales, materiales e inmateriales (intrínsecamente relacionados, sobre todo por el poder sensible y simbólico de lo físico), dan lugar a un flujo de servicios de mayor valor que suponen su fuente de retorno económico fundamental y entre los cuales el turismo juega un papel preponderante (Alonso y Martín, 2013). Soslayar la dimensión económica del Patrimonio Cultural desde posiciones estrictamente conservacionistas, contribuye a debilitar su imprescindible preservación. Mientras que aprovecharlo de manera sostenible y responsable supone una oportunidad de contribuir a mejorar las condiciones de vida de las personas, tanto en lo material (riqueza, empleo, innovación, emprendimiento), como en lo inmaterial (identidad, participación, formación, satisfacción, disfrute, calidad de vida, etc.) (Carta de Bruselas 2009).

También respecto de la actividad turística la importancia de la sostenibilidad ha quedado plasmada en la literatura científica. Son de sobra conocidos los impactos ambivalentes del turismo (Jurowski y Gursoy, 2004; Mason, 2008; UNWTO, 2013), entre los que la turistificación es actualmente uno de los más destacados, negativamente. La turistificación, entendida como el "impacto que tiene para el residente de un barrio o ciudad el hecho de que los servicios, instalaciones y comercios pasen a orientarse y concebirse pensando más en el turista que en el ciudadano que vive en ellos permanentemente" (Fundéu, 2018), hace énfasis en los agentes externos más que en los internos. Cuando lo que se viene definiendo como turismo sostenible es el hecho de que "Una ciudad, (que) pueda vivir con el turismo y no solamente de turismo" (Milano, Cheer y Novelli, 2019), respetando y conservando las distintas dimensiones urbanas.

La necesaria sostenibilidad del turismo fue puesta de relevancia con la celebración de la Comisión Mundial sobre Medio Ambiente y Desarrollo en 1987 (Informe Brundtland) y desde entonces se ha ido consolidando la postura de que no sólo importa el desarrollo económico, sino que hay que compatibilizarlo con el respeto medioambiental y social (Elkington, 2002). Es lo que posteriormente ha ido derivando hacia el turismo responsable (Declaración de Ciudad del Cabo, 2002), el turismo verde o la utopía del turismo sostenible (Pulido y Pulido, 2015; Aronsson, 2000).

Pese a esa orientación, a menudo la sostenibilidad turística parece ser un compromiso más retórico que real (Aronsson, 2000; Liu, 2003; Saarinen, 2006; Weaver, 2009; Rull, 2010), debido a la dificultad de su garantía en las distintas intervenciones (Becker, 2013; McCool et al, 2013). Ya en la Conferencia de Naciones Unidas sobre el Desarrollo Sostenible (Río de Janeiro 2012), se reconocía la dificultad de avanzar por ese camino y se planteaban soluciones por la vía de la Economía Verde (Pulido y Pulido, 2015; Stone, 2013). Se planteaba como uno de los principales objetivos el asegurar un compromiso político renovado para el desarrollo humano sostenible (Ehrlich, 2009). Bramwell (2015: 204) recuerda que el turismo sostenible se ha vinculado tradicionalmente con "la preservación de los ecosistemas, la promoción del bienestar humano, la equidad inter e intergeneracional, y la participación pública en la toma de decisiones". Se trata de que los agentes excluidos de la gestión del turismo y el patrimonio cobren un papel relevante, contribuyendo al enriquecimiento de las visiones y al ejercicio del derecho a la ciudad de las personas que la viven, sustanciados en el derecho a la participación y apropiación de la ciudad (Lefebvre, 1969; Fenster, 2010; Buckingham, 2011). 
Si es cierto que el desarrollo económico es uno de los resultados de la puesta en valor del Patrimonio Cultural $^{5}$, en ocasiones la saturación turística genera la turismofobia de los residentes (Navarro, 2015) y otros efectos negativos sobre la comunidad local (Calle, 2019). En lo sociocultural, la conflictividad entre residentes y turistas, la pérdida de idiosincrasia de la cultura receptora, de identidad local y del valor patrimonial de lo local (Sánchez, 2012; Crespi y Domínguez, 2016). De ahí la importancia para el futuro del turismo de tomar en consideración a la población local, adaptarse a los cambios y comprometerse con la sostenibilidad (Prats, 2009; Huete, 2010; García, 2019; Domínguez, 2019).

Mientras tanto, el aprovechamiento social de los bienes culturales para dotar de mayor calidad de vida a las personas se hace cada vez más presente (Florida, 2009; Hania et al, 2012; Alonso y Martín, 2013; Pérez y Lois, 2018). Aspectos como la imagen externa que proyectan los territorios son considerados también como un activo económico, social y político (Zukin, 1995; Beck, Giddens y Lash, 1997; Nyseth y Sognnaes, 2013) para la identidad urbana. Mediante estrategias de promoción, se potencia la consolidación del sentimiento de pertenencia, asociado al orgullo y la calidad de vida percibida, lo cual permite una mayor satisfacción de los ciudadanos e implicación en la vida colectiva (Radoslav, Branea y Gaman, 2013). En este marco, la preservación y promoción del Patrimonio Cultural como condensación de la historia también incrementa la mejora de la imagen de los agentes públicos y privados responsables, que experimentan una mayor legitimación social de sus actuaciones (Marcos, 2004; Dormaels, 2011; Preece, 2020). Lejos de la gestión excluyente del Patrimonio y lo urbano -desde arriba- se han ido proponiendo nuevas líneas que dan cabida a la participación ciudadana que favorece el empoderamiento de aquella y la legitimidad de las políticas públicas.

Esta línea es la que se viene poniendo de manifiesto en las actuales tendencias de los estudios sobre patrimonio (Castillo, 2015; ICOMOS, 2017) y donde se inscribe este estudio. Los resultados obtenidos han sido útiles para hacer una primera aproximación a los cambios necesarios en el tratamiento del Patrimonio Cultural de estas tres localidades cercanas a la ciudad de Madrid, a fin de armonizar, su necesaria preservación con un mejor aprovechamiento social y político y su sostenibilidad como destino turístico responsable. Supone una contribución de base práctica y empírica, a los estudios sobre Patrimonio Cultural y sostenibilidad, que puede revertir en una mejor gestión y que, de manera evidente, pueden ser de utilidad en otros escenarios con realidades parecidas.

\section{Objetivos y metodología}

Como ya se ha planteado, el problema de partida es el de los impactos negativos del turismo en la gran ciudad (densidad turística, ruido, airbnbización urbana, saturación, pérdida de identidad, etc.), como el caso del municipio de Madrid, que lo hacen insostenible en varias dimensiones. El objetivo es analizar la posibilidad y potencialidad de difundir el turismo más allá de los confines de Madrid municipio, para redistribuir sus impactos en aras de una mayor sostenibilidad regional. Prestamos especial atención a un activo hasta hace poco no considerado, como es la ciudadanía de dichos espacios, capaz de proporcionar una información de gran relevancia para la estrategia a desarrollar. El objeto de análisis elegido está conformado por tres lugares de gran riqueza patrimonial: Alcalá de Henares, Aranjuez y San Lorenzo de El Escorial, que sirven como arena de investigación. A ellos habría que añadir, a partir de ahora (julio de 2021), el eje Prado-Retiro, que viene a reforzar el papel central de la capital en el escenario patrimonial regional.

Una vez problematizado el estudio, se hace un diagnóstico de la actividad turística de dichas localidades, que son visitadas principalmente por su patrimonio cultural, pero desde una visión clásica y conservadora del atractivo turístico, pese a contar con atractivos adicionales.

En segundo lugar y debido a la ausencia de estudios locales, se desplegó una metodología ad hoc: documental y de redes, cualitativa (grupos de discusión y entrevistas), cuantitativa (encuestas y sondeos) y con procesos participativos, para indagar acerca de las preferencias y actitudes de agentes implicados y dar voz a la base social para la gestión urbana. En síntesis, la metodología de estudio se conformó por cuatro herramientas complementarias:

a) Análisis documental: científico-técnico, de guías de turismo, turoperadores y redes sociales.

b) Metodología cualitativa: entrevistas en profundidad a responsables de la gestión patrimonial y turística regional. Posteriormente se celebraron tres grupos de discusión, uno en cada municipio, con representantes locales de las administraciones a nivel político y técnico, del empresariado vinculado a la actividad turística y de las asociaciones culturales. 
c) Metodología cuantitativa: aplicación de encuestas a residentes y visitantes. Residentes: 585 cuestionarios en las tres localidades ${ }^{6}$, Alcalá de Henares (196), Aranjuez (196) y San Lorenzo de El Escorial (193). Turistas: 297 cuestionarios estructurados ${ }^{7}, 99$ en cada municipio.

d) Metodología participativa: procesos participativos en las tres ciudades que fomentó las relaciones entre agentes (habitantes y visitantes) y proporcionó información sobre atractivos potenciales.

Tabla 1: Ficha técnica de entrevistas en profundidad, grupos de discusión y procesos participativos

\begin{tabular}{|c|c|c|}
\hline \multicolumn{3}{|c|}{ Entrevistas en profundidad } \\
\hline Organismo responsable & Fecha de celebracion & Responsable \\
\hline E1 Comunidad de Madrid & 3 marzo 2015 & $\begin{array}{l}\text { Dirección General de Patrimonio } \\
\text { Histórico }\end{array}$ \\
\hline E2 Comunidad de Madrid & 4 marzo 2015 & $\begin{array}{l}\text { Subdireccion General de Desarrollo y } \\
\text { Sostenibilidad Turística }\end{array}$ \\
\hline $\begin{array}{l}\text { E3 Admon. General del } \\
\text { Estado - Patrimonio Nacional }\end{array}$ & 10 abril 2015 & $\begin{array}{l}\text { Dirección de Inmuebles y Medio } \\
\text { Natural }\end{array}$ \\
\hline \multicolumn{3}{|c|}{ Grupos de Discusión } \\
\hline Ciudad Patrimonio & Fecha de celebracion & Participantes \\
\hline G1 Alcala de Henares & 26 mayo 2015 & \multirow{3}{*}{$\begin{array}{l}\text { Técnicos de la Administración Local y } \\
\text { agentes públicos y privados relacionados } \\
\text { con la gestión patrimonial y turística }\end{array}$} \\
\hline G2 San Lorenzo de el Escorial & 27 mayo 2015 & \\
\hline G3 Aranjuez & 28 mayo 2015 & \\
\hline \multicolumn{3}{|c|}{ Acciones Participativas } \\
\hline Ciudad Patrimonio & Fecha de celebracion & Participantes \\
\hline A1 San Lorenzo de el Escorial & 1 y 10 octubre 2015 & $\begin{array}{l}\text { Encuentro-taller con asociaciones y } \\
\text { acción en polideportivo municipal }\end{array}$ \\
\hline A2 Aranjuez & 10 octubre 2015 & Acción en Plaza de la Constitución \\
\hline A3 Alcala de Henares & 28 noviembre 2015 & Acción en Plaza Cervantes \\
\hline
\end{tabular}

Fuente: elaboración propia.

Figura 2: Fotografías de los procesos participativos (Alcalá de Henares y San Lorenzo de El Escorial)
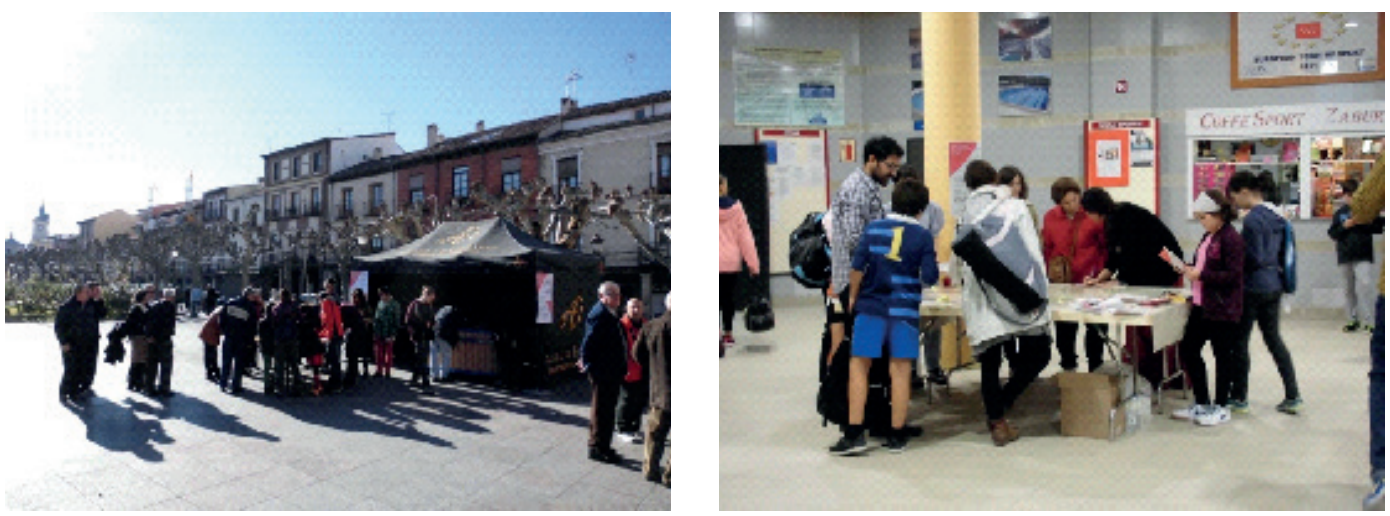

Fuente: Autoría de la empresa Paisaje Transversal (colaborador en el estudio referenciado) 
Gracias al planteamiento metodológico dinámico y triangulado, las distintas fases del desarrollo de la investigación (estudio documental, de redes, entrevistas a responsables, grupos de discusión, etc.) fueron aportando resultados que sirvieron a su vez para perfilar el diseño del análisis de demanda turística (determinación del momento y los lugares de ejecución de las encuestas; selección de dimensiones y variables; redacción de cuestionarios).

\section{Resultados: el turismo en lugares patrimoniales de la periferia madrileña}

En nuestra investigación, obtuvimos información de primera mano sobre la actitud de la población hacia el turismo y su incremento, el perfil del turista, la identificación de potenciales recursos de atracción más allá del Patrimonio tradicional y la puesta en valor de otras áreas que fortalecen la identidad urbana.

\subsection{La visión de los residentes como valor}

En la consulta a residentes se ponía de relieve el valor del Patrimonio entre la población y la potencialidad del atractivo local. Los ciudadanos, a la hora de valorar los elementos clave para la marcha de la economía local, coincidían en señalar al turismo y al Patrimonio Cultural (8,2 puntos sobre 10) como motores activos de sus municipios (Gráfico 1). Una imagen de clara de vinculación del desarrollo económico de estos municipios con el turismo cultural, con la puesta en valor del Patrimonio y con Madrid ciudad como nodo, mercado turístico prioritario y fuente de sinergias para la atracción de visitantes.

\section{Gráfico 1: Valoración de elementos clave para la economía local, promedios}

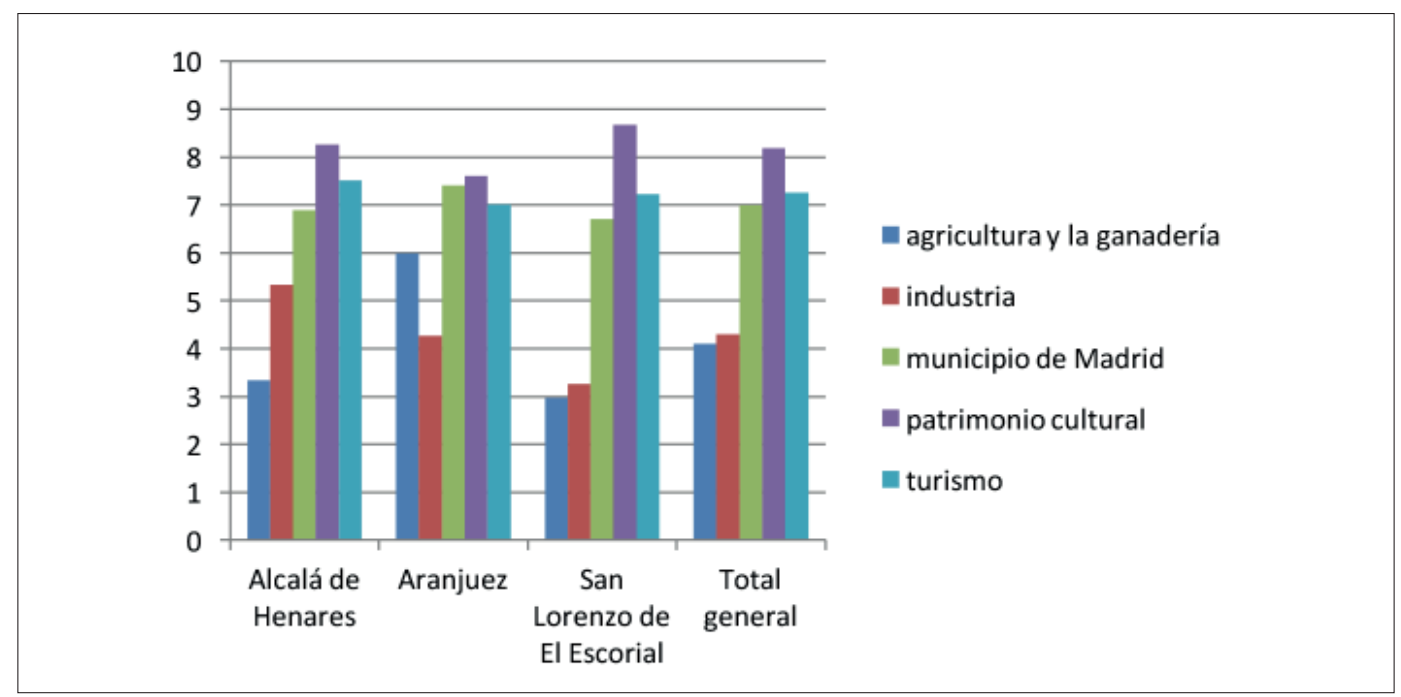

Fuente: Elaboración propia a partir de las encuestas a residentes.

Además, este posicionamiento hacia el turismo cultural como motor de la economía local tiene recorrido en las tres ciudades y no denota excesivo problema en cuanto al impacto de saturación turística en ellas. Esta cuestión importante y complicada, no parece ser un problema en estas tres localidades. De hecho, la mayoría de encuestados califica el número de visitantes como aceptable $(47,6 \%$ del total) y otros muchos desearían recibir aún más (el 34\% los considera insuficientes), siendo marginales los casos que los consideran excesivos. Así pues, el turismo cultural es considerado un sector relevante para el desarrollo local, ante el que la población muestra una actitud positiva y con cierta capacidad para acoger a un mayor número de turistas.

\subsection{Cómo son los turistas: realidad y potencialidades}

A continuación, se abordaba el estudio de las actitudes de los turistas en dichos municipios para poder abrir el campo de interés del turismo sobre estos espacios en aras de desarrollo local más allá del 
Patrimonio. En un primer lugar se define el perfil de los turistas a partir de la información primaria que aporta este estudio para entender quién y cómo es este público target de las campañas de atracción.

En el caso del turismo cultural, se habla de la atracción de turistas, la experiencia de las visitas, la atención coordinada y con perspectiva global, la oferta, en suma, de un producto integral y adaptado a la demanda. Y es que, pese a ser una cuestión vital siempre que se hable de la demanda de cualquier mercado, demasiado a menudo en la gestión turística y cultural, predomina una aproximación basada en la experiencia de los agentes implicados, mientras que sólo se dispone, en el mejor de los casos, de información parcial sobre los demandantes. A menudo dicha información proviene de las oficinas de turismo o de los inmuebles visitados, pero se carece de cifras detalladas a nivel municipal sobre el perfil de los turistas, la satisfacción con su experiencia, sus demandas o su relación con las poblaciones locales. Como se verá, esta situación también afecta de manera significativa a los tres destinos estudiados.

La muestra de turistas de las tres localidades presenta perfiles similares, componiéndose principalmente de un público de edades intermedias (la mitad de entre 40 y 64 años), equilibrado en cuanto a hombres o mujeres, con nivel de estudios medio y medio alto, y que mayoritariamente trabajaba ( $70 \%$ de ocupados). Un elemento a tener muy en cuenta es la importancia del turismo nacional para las tres ciudades, pero sobre todo en Aranjuez, donde casi el 90\% de los encuestados residía en España. Que se trate en gran medida de destinos turísticos fundamentalmente nacionales, tiene importantes implicaciones, tanto positivas como negativas. Más aún si tenemos en cuenta que el $85 \%$ de los visitantes españoles de Aranjuez, el $75 \%$ de los de El Escorial y el 68\% de Alcalá de Henares, son de la región madrileña, y de entre éstos, fundamentalmente con origen en la ciudad de Madrid, confirmando su importancia como atractivo turístico de referencia, pero también algunos condicionantes importantes derivados de la cercanía de sus domicilios (los tres destinos se sitúan a 35-55 kilómetros de la ciudad de Madrid). Consiguientemente se cuenta con un turista de origen regional, madrileño en gran medida, y que se corresponde con un perfil de edad mediana, con titulación media y que trabaja principalmente.

\subsection{El atractivo turístico del Patrimonio Cultural}

Se comprueba a continuación cómo es la estancia media del turista tipo de estos tres lugares, evidenciándose que la mayoría de los visitantes no pernocta en estas localidades. Esta situación es especialmente acusada en Aranjuez, donde sólo el 15\% de los encuestados no era excursionistas ${ }^{8}$. Otra cuestión destacada es que la mayor parte de esa minoría de turistas que sí pernocta, no lo hace en estas tres localidades (como media, sólo uno de cada tres de ellos), sino sobre todo en la ciudad de Madrid.

Esa situación condiciona de manera necesaria otro aspecto clave: la duración de las visitas, aspecto fundamental para la demanda de servicios y el volumen final de gasto turístico. El 90\% de encuestados sólo pasa medio día en esas ciudades y apenas un 6\% permanece más de un día en ellas. También se extrae información interesante al atender al tamaño del grupo turístico, observándose que Aranjuez destaca como destino de viajes en pareja y también El Escorial, aunque en menor medida, mientras que Alcalá presenta un mayor volumen de viajes grupales (familias, organizados, etc.).

Tabla 2: Características del viaje (porcentajes y escala Likert)

\begin{tabular}{|l|c|c|c|c|}
\hline & $\begin{array}{c}\text { Alcalá de } \\
\text { Henares }\end{array}$ & Aranjuez & $\begin{array}{c}\text { San Lorenzo } \\
\text { del Escorial }\end{array}$ & Total \\
\hline Duración en número de días \\
\hline 1 & $55,56 \%$ & $84,85 \%$ & $65,66 \%$ & $68,69 \%$ \\
\hline 2 & $22,22 \%$ & $9,09 \%$ & $14,14 \%$ & $15,15 \%$ \\
\hline 3 & $6,06 \%$ & $3,03 \%$ & & $3,03 \%$ \\
\hline Más de 3 & $16,16 \%$ & $2,02 \%$ & $20,20 \%$ & $12,80 \%$ \\
\hline No contesta & $1,01 \%$ & & $0,34 \%$ \\
\hline Lugar de pernoctación & & & \\
\hline Esta ciudad & $36,36 \%$ & $53,33 \%$ & $23,53 \%$ & $34,41 \%$ \\
\hline Madrid & $52,27 \%$ & $40,00 \%$ & $52,94 \%$ & $50,54 \%$ \\
\hline
\end{tabular}




\begin{tabular}{|c|c|c|c|c|}
\hline & $\begin{array}{l}\text { Alcalá de } \\
\text { Henares }\end{array}$ & Aranjuez & $\begin{array}{l}\text { San Lorenzo } \\
\text { del Escorial }\end{array}$ & Total \\
\hline $\begin{array}{l}\text { Otra ciudad en la Comunidad de } \\
\text { Madrid }\end{array}$ & $11,36 \%$ & $6,67 \%$ & $20,59 \%$ & $13,98 \%$ \\
\hline Otros & & & $2,94 \%$ & $1,08 \%$ \\
\hline \multicolumn{5}{|l|}{$\begin{array}{l}\text { Duración de la visita a la } \\
\text { ciudad }\end{array}$} \\
\hline Medio día & $80,81 \%$ & $90,82 \%$ & $91,92 \%$ & $87,84 \%$ \\
\hline 1 día & $7,07 \%$ & $6,12 \%$ & $4,04 \%$ & $5,74 \%$ \\
\hline 2 días & $3,03 \%$ & $2,04 \%$ & $3,03 \%$ & $2,70 \%$ \\
\hline 3 días & $6,06 \%$ & & & $2,03 \%$ \\
\hline Más de 3 días & $3,03 \%$ & $1,02 \%$ & $1,01 \%$ & $1,69 \%$ \\
\hline \multicolumn{5}{|l|}{$\begin{array}{l}\text { Número de personas con las } \\
\text { que viaja }\end{array}$} \\
\hline Solo & $19,19 \%$ & $22,45 \%$ & $16,33 \%$ & $19,32 \%$ \\
\hline 2 personas & $24,24 \%$ & $51,02 \%$ & $43,88 \%$ & $39,66 \%$ \\
\hline 3 a 9 personas & $48,48 \%$ & $22,45 \%$ & $31,63 \%$ & $34,24 \%$ \\
\hline 10 o más personas & $8,08 \%$ & $4,08 \%$ & $8,16 \%$ & $6,78 \%$ \\
\hline \multicolumn{5}{|l|}{ Como conoció el sitio } \\
\hline Agencia de viajes & $1,01 \%$ & & $3,03 \%$ & $1,35 \%$ \\
\hline Amigos/conocidos/familia & $63,64 \%$ & $24,24 \%$ & $42,42 \%$ & $43,43 \%$ \\
\hline Internet & $6,06 \%$ & $5,05 \%$ & $6,06 \%$ & $5,72 \%$ \\
\hline Prensa/radio/TV & $1,01 \%$ & & $4,04 \%$ & $1,68 \%$ \\
\hline Publicidad & $2,02 \%$ & & & $0,67 \%$ \\
\hline Otros & $26,26 \%$ & $67,68 \%$ & $42,42 \%$ & $45,45 \%$ \\
\hline No sabe / No contesta & & $2,03 \%$ & $2,02 \%$ & $1,68 \%$ \\
\hline \multicolumn{5}{|l|}{$\begin{array}{l}\text { Motivos de elección del destino } \\
\text { (promedio escala Likert 1-5) }\end{array}$} \\
\hline Patrimonio cultural & 4,64 & 4,51 & 4,70 & 4,62 \\
\hline Actividades culturales & 3,09 & 2,31 & 2,36 & 2,63 \\
\hline Ocio (gastronomía, compras, etc.) & 3,84 & 3,59 & 3,39 & 3,61 \\
\hline Entorno natural & 3,09 & 4,35 & 4,56 & 4,00 \\
\hline Cercanía al domicilio & 3,18 & 3,68 & 3,52 & 3,46 \\
\hline Paquete turístico & 1,28 & 1,42 & 1,21 & 1,30 \\
\hline
\end{tabular}

Fuente: Elaboración propia a partir de resultados de las encuestas a turistas.

En cuanto a la vía de conocimiento de estos destinos que motivó la decisión de su visita, se puso de manifiesto el bajo impacto de la difusión turística vía Internet, medios de comunicación o publicidad (sólo alrededor de un 8\% del total conocieron la existencia de esos destinos gracias a estas vías) o de las agencias de viajes (indicativo de un mayor grado de auto-organización y pérdida de peso de los turoperadores); y sin embargo, el alto impacto del boca a boca (amigos, conocidos, familiares, etc.), junto a otros factores muy diversos (por cercanía, por ser conocidas desde siempre, por trabajo, etc.). Esta situación llama la atención, pues en el análisis cualitativo de los discursos de agentes locales se ponía énfasis en la promoción en los medios de comunicación tradicionales, lo cual demuestra la disonancia entre la difusión que los agentes creían más eficaz (y por eso se utiliza: web, publicidad, etc.) y lo que en realidad lo era (el boca a boca). Precisamente esto último abre la puerta a incorporar la visión que 
los vecinos destacaban como atractiva, más cerca del turismo experiencial y diferenciado, que del estandarizado y patrimonial. Así las aportaciones hechas desde la base social servirían de atractivos para otros sectores supralocales, tanto de la región como de otras escalas.

Por su parte, los turistas de las tres ciudades tienen claro que llegan a ellas atraídos primeramente por su Patrimonio Cultural y, en el caso de San Lorenzo de El Escorial y Aranjuez, también por su entorno natural (segundo lugar). El tercer factor de atracción es la oferta de ocio y de actividades recreativas y el cuarto la proximidad al domicilio. Por lo tanto, es evidente que el activo patrimonial es el principal atractor y que, sin embargo, la oferta cultural, de ocio, gastronómica, etc. valorizada por la población local, podría potenciarse más, puesto que aparece insuficientemente desarrollada. Es en esta área donde el estudio señalaba una de las potencialidades de desarrollo social y político al tiempo que económico. En el corto plazo, además, el escenario pandémico y -en su momento- pospandémico, refuerzan la ventaja que otorga a estos lugares su cercanía al mercado emisor madrileño sobre todo cuando las distancias se acortan.

\subsection{Cómo revierte el gasto turístico en el destino}

Puesto que supondría un indicador de éxito de las estrategias promotoras, así como fuente para el desarrollo local, también se ha incluido un apartado de estudio acerca del impacto del turismo sobre la economía local. Un primer determinante del gasto turístico es el número de personas que viajan (cuya consideración lleva al concepto de gasto per cápita) y la lejanía de su domicilio. Se comprobó que los encuestados que residían más lejos hicieron un gasto general mayor que los más cercanos, pareciendo así que la atracción de más turistas más allá del ámbito regional, podría tener un mayor impacto económico. Sin embargo, el estudio indicó que la mayoría de los visitantes provenían, como ya se ha señalado, del área española y madrileña, con un gasto total promedio de 100 euros por persona y viaje. Otro factor clave es el número de días del viaje, siendo generalmente mayor el gasto a medida que aumenta su duración global. Este elemento afecta a la cantidad diaria gastada: el 44\% de los encuestados gastaba menos de 20 euros por persona por día; un tercio, de 20 a 49 euros y el $22 \%, 50$ euros o más. Sin embargo, no toda esa cantidad se desembolsa en las ciudades estudiadas. De hecho, más del 8\% de los encuestados no incurría en ningún gasto en Aranjuez y San Lorenzo del Escorial y más de un tercio en general gastaba menos de 10 euros por persona por día. Solo el 15\% gastaba más de 50 euros por día por persona. Se hace así evidente que sólo una limitada fracción del gasto turístico acaba siendo recibido a nivel local. Pese a que se necesitan estudios de mayor alcance y representatividad, vemos que en el impacto económico del turismo sobre estas localidades el factor determinante es la duración de la visita, dado que el gasto potencial está determinado por factores que se escapan a la gestión de los agentes locales (renta de los turistas, lugar de procedencia, hábitos y preferencias individuales, etc.). En consecuencia, resulta esencial para conseguir un mayor retorno efectivo, no sólo potenciar la atracción de visitantes, sino también proponer una oferta cultural capaz de fijar al visitante en el municipio por más tiempo o más a menudo.

\subsection{Qué elementos se pueden potenciar según la ciudadanía}

Continuando con esa idea de conveniencia de enriquecer la oferta de este tipo de destinos, analizamos la experiencia de la visita con la vista puesta en la detección de recursos que muestran el atractivo real y con potencial para dinamizar el turismo. Para ello, se pidió a participantes -a través de las distintas metodologías implementadas- que propusieran una serie de lugares a visitar y de actividades a realizar en cada una de las tres ciudades. De este modo, los atractores locales para los residentes se ponían en el punto de mira de los turistas para conseguir una mayor efectividad. El propósito era hacer de los vecinos embajadores turísticos, difundiendo el atractivo de sus municipios, lo que potencia su implicación y sus sentimientos de orgullo, pertenencia e identidad, revirtiendo todo ello en beneficio del conjunto del municipio. Ya que nos movemos en marcos de turismo como experiencia, la experiencia de los locales puede ser un buen atractivo para los visitantes, y de hecho los vecinos aportaron mucha información sobre lugares y actividades atractivas que podrían ponerse en valor. La ya antigua definición del turismo cultural como conocimiento de lo físico, el monumento, etc. (Yale, 1991), queda denostada ante el turismo cultural experiencial. Este sería el punto clave para potenciar eslóganes como To live like a local:

"Pero si la gente que pernocta no le damos facilidades para tomar algo hasta tal hora no es una cosa que atraiga a la pernoctación, si no puedes salir y tener unos alicientes (...) Cuando están los cursos de verano 
viene gente y eso se nota, salen por la noche a tomar algo y se sientan en las terrazas de la peatonal, se nota se ven grupos" (G2 grupo de discusión de El Escorial)

"Lo importante es arraigar a la generación siguiente. El hospital nos hizo mucho bien porque la gente empezó a poner en el DNI Alcalá de Henares y ya empezó a haber mucha gente que se sentía de Alcalá pero cuando éramos pequeños la gente no se sentía de Alcalá y si no te sientes de Alcalá no puedes amar algo que no sientes como tuyo, para amar algo tienes que sentirlo como tuyo y entonces uno de los desafíos que tenemos es conseguir que la gente ame a la ciudad y para que ame a la ciudad debe conocerla y para conocerla debe sentirla como suya” (G1 grupo de discusión Alcalá de Henares)

El análisis de la información publicitada en redes y webs reveló un discurso promotor oficial, tradicional, estandarizado y repetitivo en contenidos, sin apenas innovación. Incluso la "marca" Patrimonio Mundial, aparecía vacía de contenido y, aunque resultaba conocida por visitantes y habitantes, era poco utilizada en estos destinos. Frente a ello, los locales proponían experiencias que iban más allá de la visión clásica, para incluir experiencias que aludían a significados diferentes de los lugares. En los procesos participativos se registraron amplios listados de sugerencias y se plantearon rutas de ocio sobre los mapas, para componer una agenda experiencial y de actividades más atractiva:

"En el momento de la salida del sol en septiembre, me encanta el reflejo del sol en las ventanas del Monasterio. Refleja una bola de fuego"; "La silla de Felipe II es un lugar de culto y mágico de la antigüedad. Es el sitio donde da el último rayo de sol del día». (A1 Proceso participativo de vecinos de San Lorenzo del Escorial). "Jugaba al fútbol enfrente del Palacio; "la plaza elíptica era un campo de fútbol, las porterías eran los plátanos. Encajaban el balón en el palacio Real” (A2 Proceso participativo de vecinos de Aranjuez).

"La plaza Cervantes era de barro, por un lado paseaban las mujeres y por otro los hombres" (A3 Proceso participativo de vecinos de Alcalá de Henares).

Así, por ejemplo, sugerían incluir en las visitas zonas ubicadas fuera de los casos históricos, como los yacimientos arqueológicos de Alcalá de Henares; o la difusión de otros valores, como la sostenibilidad con aparcamientos disuasorios o la recomendación del transporte público para la visita; o la propuesta de un turismo de naturaleza (disfrute de entornos naturales, paseos en bicicleta, etc.) o experiencial (barco en el río en Aranjuez, experiencia enológica, etc.); o la elaboración de mapas de atractivo para los visitantes (rutas gastronómicas, guías de los mejores bares y restaurantes, qué hacer un viernes o en familia, turismo con niños, etc.). También se recogieron propuestas que iban más allá del ámbito municipal, como la coordinación con otros enclaves turísticos cercanos para promocionar áreas de desconcentración en lugar de incidir en la atracción sobre un solo punto. Este tipo de medidas requeriría la coordinación de los agentes institucionales en sus diferentes escalas territoriales (nacional, regional, local), aspecto que en la actualidad se evidencia relativamente descoordinado. Un buen ejemplo en tal sentido es una estrategia comunicacional coordinada entre la ciudad de Madrid y los tres sitios, propuesta que emergió en distintos momentos de la investigación. Entendemos que la declaración del Paseo del Prado y el Parque del Retiro como nuevo sitio Patrimonio Mundial en julio de 2021, es una oportunidad añadida en esa misma línea.

\section{Discusión}

Comenzábamos este trabajo partiendo del problema actual de los impactos negativos del turismo, en particular para las ciudades: sobreexplotación de recursos, pérdida de calidad de vida de los residentes, merma de la experiencia turística, etc. (Barrado e Hidalgo, 2019). Los destinos basados en el Patrimonio Cultural, como son los analizados en este trabajo, no escapan a esa situación (Troitiño, 2012), siendo en su caso menos soportable aún, ya que afecta a la naturaleza misma de esos bienes, que lleva implícita la necesidad de su sostenibilidad y la gestión responsable (UNWTO, 2013; ICOMOS, 2017).

En este estudio ha quedado demostrada la conveniencia de poner en marcha estrategias orientadas a la sostenibilidad de los destinos, mediante el esponjamiento en áreas supralocales del turismo que gravita sobre dichos espacios centrales. Para ello, es clave potenciar el atractivo de otros lugares cercanos, incrementar el tiempo de estancia de los visitantes en ellos -con el consiguiente aumento del gasto turístico- y atraer a otros sectores no interesados hasta el momento. No memos importante es la realización de estudios de público, la mejora de los sistemas de información turística y, sobre 
todo, hacer partícipe de la gestión del Patrimonio y el turismo a la población local, de manera que su conocimiento se ponga al servicio del proceso. En esa captación de mercados nuevos, destacaría como apuesta el turismo de proximidad, de gran potencial y clave en contextos como el actual, marcado en el corto y medio plazo por la pandemia de Covid-19 (Arold, 2021), en el que se restringen los movimientos de larga distancia. Ello permitiría, además, constituirse en alternativa a la ciudad de Madrid en los movimientos turísticos, aliviando la saturación de ese destino de referencia. Como venimos proponiendo, la reciente incorporación de la arteria capitalina al listado UNESCO, supone una oportunidad que se suma al puzzle actual y abre nuevas posibilidades para reforzar la capacidad de atracción del conjunto de lugares patrimoniales de una región que se consolida como referencia internacional.

De igual modo y desarrollando esa idea, en áreas supralocales donde predomina una gestión fundamentada en criterios de competencia administrativa, parcial y localista, el estudio pone en valor la necesidad de coordinar las distintas administraciones y agentes en entornos relacionales sobre áreas más amplias. Se trataría de considerar los flujos y movimientos espaciales sobre la base de áreas funcionales y no estrictamente administrativas o sectoriales. Para ello, insistimos, es imprescindible orientar en tal sentido la gestión patrimonial y turística (UNWTO, 2013), mediante la coordinación de los múltiples agentes implicados en ambas dimensiones: administraciones de distintos niveles, agentes económicos y los propios vecinos de las localidades. Los primeros tienen en su mano diseñar políticas públicas reequilibradoras del desarrollo regional, utilizando como palancas el Patrimonio y el turismo cultural. Los segundos, tienen la necesidad evidente de continuar adaptando su oferta comercial a los cambios de la demanda turística. Y la base social es un actor de primer orden con mucho que aportar para enriquecer las experiencias de los visitantes (turismo experiencial, emocional, etc.), al tiempo que su participación en esos procesos fortalece su identidad, mejora la cohesión social y contribuye a una mejor gobernanza urbana, proporcionando nuevas rentabilidades al Patrimonio y al turismo más allá de la contabilidad económica. Se consigue de ese modo alcanzar sinergias entre distintos ámbitos, sectores, colectivos, etc., de cara a una planificación integral y holística, de acuerdo a una perspectiva relacional.

Las estrategias tradicionales, de amplia tradición en la planificación turística y del patrimonio, se ponen así en cuestión por su tendencia a generar intensas inercias en el funcionamiento de las instituciones, difíciles de abandonar y que son una barrera para la implementación de planteamientos nuevos más participativos, a pesar de sus buenas intenciones (Santana, 1997; Lara de Vicente y López-Guzmán, 2004; ICOMOS, 2011; Díez et al, 2013). Dichas estrategias clásicas normalizan una planificación que va de arriba-abajo, esto es, desde las instituciones implicadas (arriba) hacia la ciudadanía y la demanda turística (abajo). En ellas el agente es la institución en sentido amplio y el objeto de las políticas es la ciudadanía y el turismo. En contraposición a esas políticas públicas e institucionales de sujeto versus objeto, planteamientos alternativos abogan por la consideración de líneas más relacionales, inclusivas, integrales y participativas, en un planteamiento de abajo-arriba y de ida y vuelta. En ellas se propone de manera efectiva perspectivas no tan jerarquizadas, que ponen énfasis en lo relacional e integral. En esta línea se destacan, por ejemplo, los trabajos de Prats, 2009; Huete, 2010; Rivera y Rodríguez, 2012; Castillo, 2015; ICOMOS, 2017; Agenda Urbana Española, 2019; Domínguez Pérez, 2019; García, 2019 y Gómez, Crespi y Domínguez, 2020.

Más aún, en este trabajo hemos constatado -en la línea de la Agenda Urbana Española (2019) y Gómez, Crespi y Domínguez (2020)- que un elemento fundamental que subyace a toda esta dinámica, es la conveniencia de potenciar la participación ciudadana en la elaboración de estrategias de turismo que pudieran revertir, tanto en el reequilibrio turístico regional, como en la mejora de la vida interna de los municipios, su identidad y gobernanza.

Por último, aparece la necesidad de mejorar los sistemas de información, promoviendo la realización de detallados estudios especializados que cuenten también con los agentes implicados, incluyendo la ciudadanía, los turistas, etc. Como ha sido reiteradamente señalado en tantos ámbitos y en particular en el del Patrimonio Cultural y el turismo (Pedersen, 2005; Comisión Europea, 2013; UNESCO, 2014; Adie y Hall, 2016; Kempiak et al, 2017; OMT, 2017), disponer de información fiable es la base que permite desarrollar estrategias de gestión adecuadas. Y es que resulta imprescindible conocer de manera efectiva dónde radican los respectivos mercados turísticos con mayor potencial, cuáles son las demandas actuales de los visitantes y su grado de satisfacción con las visitas, así como su potencialidad para abrir nuevas vías de interés. 


\section{Conclusiones}

Los casos que se han analizado en este trabajo, localizados en la periferia de la ciudad de Madrid, son un buen ejemplo del delicado escenario que aparece por el uso turístico del Patrimonio Cultural. En particular, de la necesidad de encontrar equilibrios entre las oportunidades de desarrollo económico y social y los riesgos de sobreexplotación de recursos y generación de impactos negativos sustantivos.

En nuestro acercamiento hemos encontrado, en primer lugar, tres lugares con una gran riqueza patrimonial y en los que el turismo tiene un papel preponderante en su economía, que hacen del Patrimonio Cultural y el turismo, motores de la actividad y el desarrollo locales. También con un gran potencial para contribuir, más allá de lo local, a la redistribución del impacto turístico regional, a su esponjamiento desde la concentración en la ciudad de Madrid.

Para lograr ambos objetivos, tres elementos son particularmente necesarios: 1) el establecimiento de actuaciones coordinadas de los múltiples agentes presentes en el escenario, orientadas a ese propósito concreto; 2) la incorporación activa de los residentes locales, generadora de sinergias hacia afuera (atracción turística) y hacia adentro (vertebración social) de las localidades; 3) la generación de conocimiento especializado sobre la materia.

Así lo revelan algunos de los resultados más significativos de la investigación, como la conveniencia no sólo de atraer e intentar prolongar la estancia de turistas lejanos, sino también de fomentar la reiteración de la visita de visitantes procedentes del rico mercado madrileño (estrategias de fidelización, aún más importantes en momentos como los actuales); la importancia del boca a boca en la promoción del destino, por encima de otros medios de difusión más establecidos (y caros); la conveniencia de modernizar y diversificar una imagen turística clásica-patrimonial para incorporar más elementos de cultura, ocio, gastronomía, etc. o una mayor integración con la población local, lo que ampliaría el horizonte de la oferta y renovarían el atractivo de estas ciudades, tanto para turistas como para unos ciudadanos que actúan como embajadores turísticos.

Aunque las conclusiones alcanzadas también resultan de aplicación en otros territorios que presenten una configuración semejante, los tres casos analizados resultan idóneos para poner en práctica este tipo de indicaciones, pues suscitan un gran interés turístico, sus vecinos se muestran receptivos a la recepción de visitantes y se detecta un recorrido significativo en los contenidos que se pueden ofertar. La clave parece estar, una vez más, en una gestión patrimonial y una programación cultural más abiertas y flexibles, adaptadas a la nueva realidad social y cultural. Todo ello redundará, en última instancia, en el desarrollo económico y social de los lugares con activos patrimoniales y la sostenibilidad territorial de la actividad turística a nivel regional.

\section{Bibliografía}

Adie, B. A. y Hall, C. M. (2017). "Who visits World Heritage? A comparative analysis of three cultural sites". Journal of Heritage Tourism,12(1): 67-80.

Agenda Urbana Española (2019). https://apps.fomento.gob.es/CVP/detallepublicacion.aspx?idpub=BAW061 Visitado 29 de septiembre de 2020.

Aguado, L. F., Palma, L. y Pulido, N. (2017). "50 años de economía de la cultura. Explorando sus raíces en la historia del pensamiento económico", Cuadernos de Economía, 36 (70): 197-225.

Alonso, J. y Martín, J. (2008). Conservación del Patrimonio Histórico de España. Análisis económico. Madrid: Fundación Caja Madrid.

Alonso, J. y Martín, J. (2013). “Activos culturales y desarrollo sostenible: la importancia económica del Patrimonio Cultural”, Política y Sociedad, 50 (3): 1133-1147.

Andres, J. L. (2015). "Mito y estrategias para un destino romántico: la ciudad de Teruel". Cuadernos de Turismo, 36: 39-54

Arold, P. (2021). Apuntes para la gestión del turismo en España tras la crisis sanitaria de la Covid-19. PASOS Revista De Turismo Y Patrimonio Cultural, 19(1), 189-194.

Aronsson, L. (2000). The Development of sustainable tourism. Nueva York: Continuum.

Barrado, D. e Hidalgo, C. (2019). "Golden hordes or mere barbarians? Discourses on tourism, touristification, and tourismophobia in Madrid's Lavapiés neighborhood”. Boletín de la Asociación de Geógrafos Españoles, (83). 
Barrio, M. J. y Herrero, L.C. (2014). "Evaluating the efficiency of museums using multiple outputs: evidence from a regional system of museums in Spain", International Journal of Cultural Policy, 20(2): 221-238.

Beck, U., Giddens, A. y Lash, S. (1997). Modernización reflexiva. Política, tradición y estética en el orden social moderno. Madrid: Alianza Editorial.

Becker, E. (2013). Overbooked. The exploding business of travel and tourism. New York.

Bedate, A. et al. (2004). "Economics Valuation of the Cultural Heritage. Application to four case studies in Spain", Journal of Cultural Heritage, 5(1): 101-111.

Bonet, L. (2013). "Heritage Tourism", en Rizzo, I. y Mingosa, A. (Eds.) Handbook on the Economics of Cultural Heritage (pp. 386-401). Cheltenham (UK): Edward Elgar.

Bramwell, B. (2015). "Theoretical activity in sustainable tourism research". Annals of Tourism Research, 54: $204-218$.

Buckingham, S. (2011). "Análisis del derecho a la ciudad desde una perspectiva de género". En Sugranyes, A. y Mathivet, C. Ciudades para tod@s. Por el derecho a la ciudad, propuestas y experiencias (pp. 59-64). Santiago: Hábitat International Coalition,

Calle, M. de la (2019). "Turistificación de centros urbanos: clarificando el debate". Boletín de la Asociación de Geógrafos Españoles, 83, 2829, 1-40.

Calle, M. de la; Ferreiro, E. y Mendoza, S. (2018). "Concentración y desconcentración de la actividad turística sobre el espacio urbano. Reflexiones en torno a Madrid”. Polígonos. Revista de Geografía, 30, 143-170.

Calle, M. de la, García, M. y Mínguez, M.C. (2015). "El despliegue del ocio turístico en el territorio de la Comunidad de Madrid”. Ciudad y Territorio. Estudios territoriales. Vol. XLVII, nº 184, pp. 293-308.

Carta de Bruselas (2009). Carta de Bruselas sobre el papel del Patrimonio Cultural en la Economía. Junta de Castilla y León.

Castillo, A. (Ed.) (2015). Actas del II Congreso de Buenas Prácticas en Patrimonio Mundial: Personas y Comunidades. Madrid: Universidad Complutense de Madrid.

Cavailles, C., Laurent, M., Maurin, S. y Sánchez, J. L. (2016). "Los turistas en el centro histórico de Salamanca: tránsito, percepción y (des)conocimiento", Cuadernos de Turismo, 37: 37-67.

Comisión Europea (2013). Herramienta del Sistema Europeo de Indicadores Turísticos para Destinos Sostenibles. Bruselas: D. G. Empresa e Industria.

Cortijo, E. y Pulido, M. (2016). "Percepción de la población residente en Cañamero acerca del geoparque Villuercas-Ibores-Jara (Extremadura, España)", Cuadernos de Turismo, 37: 93-112.

Crespi Vallbona, M. y Domínguez Pérez, M. (2016). "Food markets and tourist cities". PASOS: Revista de Turismo y Patrimonio Cultural, 14(2), 401-416.

Devesa, M. et al. (2012). "Repercusiones económicas y sociales de los festivales culturales. El caso el Festival Internacional de Cine de Valdivia”, EURE - Revista Latinoamericana de Estudios Urbano-Regionales, 38: 95-115.

Díez, J. I. M., Gutiérrez, R. R. \& Pazzi, A. (2013). “De arriba hacia abajo o de abajo hacia arriba?: un análisis crítico de la planificación del desarrollo en América Latina”, Geopolítica(s), vol. 4, núm. 2, 199-235.

Domínguez Pérez, M. (2019). "Del patrimonio como la cristalización de la historia de la comunidad urbana y su identidad”, PH Revista Patrimonio Histórico, 97. Instituto de estudios andaluces.

Dormaels, M. (2011). "Patrimonio, patrimonialización e identidad. Hacia una hermenéutica del patrimonio". Revista Herencia, 24 (1-2), 7-14.

Ehrlich, P. E. (2009). "Cultural evolution and the human predicament". Trends in Ecology \& Evolution, 24: 409-412.

Elkington, J. (2002). Cannibals with forks: the triple bottom line of 21st century business (Reprint). Capstone.

Encuesta de Ocupación Hotelera (2020). INE https://www.ine.es/dyngs/INEbase/es/operacion.htm?c=E stadistica_C\&cid $=1254736177015 \&$ menu $=$ resultados\&secc $=1254736195376 \& i d p=1254735576863 \#$ !tabs-1254736195376 visitado 29 de septiembre de 2020

European Commission (2012). Promoting cultural and creative sectors for growth and jobs in the EU. Brussels.

Fenster, T. (2010). "El derecho a la ciudad y la vida cotidiana basada en el género", en Sugrañes, A. y Mathivet, C. Ciudades para tod@s: por el derecho a la ciudad, propuestas y experiencias (pp. 65-80). Santiago de Chile: HIC.

Florida, R. (2009). Ciudades creativas. Barcelona, Paidós. 
Frey, B. y Steiner L. (2011). "Correcting the imbalance of the Word heritage list: did the UNESCO strategy work?", Journal of International Organizations Studies, 3(1): 25-40.

FUNDEU (2018). Diccionario. (Fundación patrocinada por la Agencia Efe y el BBVA, asesorada por la RAE). https://www.fundeu.es/recomendacion/turistificacion-neologismo-valido/ visitado 8 de octubre 2020

García, M., De la Calle, M. y Yubero, C. (2017). "Cultural Heritage and Urban Tourism: Historic City Centres under Pressure”. Sustainability 9: 1346.

García, M., de la Calle, M. y Mínguez, M. (2015). "Cultura y Patrimonio en las prácticas turísticas de la población madrileña”, International Journal of Scientific Management and Tourism, 4: 97-124.

García, R. (2019). La Sostenibilidad, clave para el futuro de la industria turística. Fecha de consulta: 5 de septiembre de 2019. https://bit.ly/2ZKwyod

Gómez, M., Crespi, M. y Domínguez, M. (2020). "El patrimonio urbano como intersección de espacio y sociedad. Una oportunidad para el replanteamiento del rol del patrimonio en las agendas urbanas", en Alonso, M. R., De Gregorio, S. y González, M. Las agendas urbanas y el gobierno de las ciudades: transformaciones, desafíos e instrumentos. Barcelona: Reus.

Hania, U., Azzadinaa, I., Sianipara, C., Setyagunga, E. e Ishii, T. (2012). "Preserving cultural heritage through creative industry: A lesson from Saung Angklung Udjo”, Procedia Economics and Finance, 4: 193-200.

Herrero, L. C. y Gómez, M. (2017). "Cultural resources as a factor in cultural tourism attraction: Technical efficiency estimation of regional destinations in Spain", Tourism Economics, 23 (2): 260-280.

Herrero, L.C. et al. (2006). "The economic impact of cultural events: a case-study of Salamanca 2002, European Capital of Culture", European Urban and Regional Studies, 13 (1): 41-57.

Huete, R. (2010). Opiniones y actitudes ante el turismo residencial en el sur de la Comunidad Valenciana http://www.theeconomyjournal.com/texto- diario/mostrar/591498/saturacion-destinos-turisticos-barcelona-exporta-modelo-sur.

ICOMOS (2011). Declaración de París sobre el Patrimonio como motor de desarrollo. Paris, UNESCO.

ICOMOS (2017). Delhi Declaration on Heritage and Democracy. Paris, UNESCO.

Informe Brundtland (1987). Nueva York: ONU.

Jurowski, C. y Gursoy, D. (2004). "Distance effects on residents; attitudes toward tourism". Annals of Tourism Research, 31(2): 296-312.

Kempiak J., Hollywood, L., Bolan, P. y McMahon-Beattie, U. (2017). "The heritage tourist: an understanding of the visitor experience at heritage attractions", International Journal of Heritage Studies, 23(4): 375-392.

Landriani, L. y Pozzoli, M. (2014). Management and Valuation of Heritage Assets. A Comparative Analysis Between Italy and USA. Springer International Publishing.

Lara de Vicente, F. y López-Guzmán, T. (2004). "El turismo como motor de desarrollo económico en ciudades Patrimonio de la Humanidad". Pasos 2(2): 243-256.

Lefebvre, H. (2017). El derecho a la ciudad, Madrid: Capitán Swing.

Liu, Z. (2003). "Sustainable tourism development: A critique". Journal of Sustainable Tourism, 11(6), 459-475.

López-Guzmán, T. y Sánchez, S. M. (2009). “Turismo comunitario y generación de riqueza en países en vías de desarrollo. Un estudio de caso en El Salvador". REVESCO. Revista De Estudios Cooperativos, 99: 85 - 103.

Manero, F. (2011). "Patrimonio cultural y transformación urbana: ordenación espacial y proyección turística de los yacimientos arqueopaleontológicos de Atapuerca (Burgos)". Cuadernos de Turismo, 27: 617-640.

Marcos, J. (2004). "La tradición, el patrimonio y la identidad”. Revista de estudios extremeños, 60 (3): 925-956.

Martos Molina, M. (2012). "Las ciudades patrimoniales en el mercado turístico cultural. Úbeda y Baeza". Gran Tour: Revista de Investigaciones Turísticas, 6: 32-82.

Mason, P. (2008). Tourism impacts Planning and management. Londres: Butterworth-Heinemann.

McCool, S., Butler, R., Buckley, R., Weaver, D. y Wheller, B. (2013). "Is Concept of Sustainability Utopian: Ideally Perfect but Impracticable?” Tourism Recreation Research, 38(2): 213-242.

Milano, C., Novelli, M. y Cheer, J. M. (2019). "Overtourism and Tourismphobia: A Journey Through Four Decades of Tourism Development, Planning and Local Concerns", Tourism Planning \& Development, $16: 4,353-357$ 
Mínguez, M.C. (2007). "Planificación y Gestión Turística en destinos patrimoniales: el caso de San Lorenzo de El Escorial (Comunidad de Madrid)". Anales de la Geografía de la Universidad Complutense de Madrid, n ${ }^{\circ}$ 27. Pp. 83-102.

Navarro, E. (2015). "La saturación de los destinos turísticos; Barcelona exporta su modelo al sur". The Economy Journal.

Nyseth, T. y Sognnaes, J. (2013). "Preservation of old towns in Norway: Heritage discourses, community processes”, Cities, 31: 69-75.

Organización Mundial del Turismo (OMT) (2017). Compendium of Tourism Statistics. Data 2011- 2015. OMT.

Palma, L. A. y Aguado, L. F. (2010). "Economía de la Cultura. Una nueva área de especialización de la Economía", Revista de Economía Institucional, 12 (22): 129-165.

Pedersen, A. (2005). Gestión del turismo en sitios del Patrimonio Mundial: Manual práctico para administradores de sitios del Patrimonio Mundial. Centro del Patrimonio Mundial, UNESCO.

Pérez, Y. (2015). "Diseño de un Sistema Integral de Análisis del Turismo (SIAT) en destinos patrimoniales", Investigaciones Turísticas, 10: 57-75.

Pérez, Y. y Lois, R. C. (2018). "Sustainability and visitor management in tourist historic cities: the case of Santiago de Compostela, Spain”. Journal of Heritage Tourism 13 (6): 489-505.

Perkumiene, D. y Pransküniené, R. (2019). “Overtourism: Between the Right to Travel and Residents' Rights". Sustainability, 11(7), 21-38.

Prats, F. (2009). "La evaluación integrada de la sostenibilidad en destinos turísticos". Conferencia Internacional de Turismo. El conocimiento como valor diferencial de los destinos turísticos. Málaga.

Preece, J. (2020). "Belonging in working-class neighbourhoods: dis-identification, territorialisation and biographies of people and place". Urban Studies Vol. 57 (4), 827-843.

Pulido, J. I. y Pulido, M. C. (2015). “Sigue vigente el paradigma del turismo sostenible? Reflexiones a la luz de la literatura reciente". Pasos. Revista de Turismo y Patrimonio Cultural, 13(6), 1315-1335.

Radoslav, R., Branea, A. M. y Gaman, M. S. (2013). "Rehabilitation through a holistic revitalization strategy of historical city centres - Timisoara, Romania", Journal of Cultural Heritage, 14 (3): e1-e6.

Rengifo, J. L., Campesino, A. J. y Sánchez, J. M. (2015). "El turismo en la ciudad de Cáceres (1986-2010): un cuarto de siglo emblemático". Boletín de la Asociación de Geógrafos Españoles, 67: 375-401.

Rivera, M. y Rodríguez, L. (2012). "Turismo responsable, sostenibilidad y desarrollo local comunitario". Córdoba (España): Cátedra Intercultural, Universidad de Córdoba.

Rizzo, I. y Mignosa, A. (Eds.) (2013). Handbook on the Economics of Cultural Heritage. Cheltenham (UK): Edward Elgar.

Rull, V. (2010). "El mito del desarrollo sostenible". Collectanea Botanica, 29: 103-109.

Saarinen, J. (2006). "Traditions of sustainability in tourism studies". Annals of Tourism Research, 33(4): 1121-1140.

Sánchez Carretero, C. (2012). "Hacia una antropología del conflicto aplicada al patrimonio". En Santamarina, B. (Ed.) Geopolíticas patrimoniales: De culturas, naturalezas e inmaterialidades. Una mirada etnográfica (pp. 195-210). Valencia: Editorial Germania, Colección Antropológicas, Valencia.

Santana, A. (1997). Antropología y Turismo, ¿Nuevas hordas, viejas culturas? Barcelona, Ariel.

Snowball, J. D. (2013). "The economic, social and cultural impact of cultural heritage: methods and examples", en Rizzo, I. y Mingosa, A. Handbook on the Economics of Cultural Heritage (pp. 438-455). Cheltenham (UK): Edward Elgar.

Stabler, M. (1997). Tourism and sustainability: Principles and Practice. Oxon: CABI.

Stone, S. (2013). "Los beneficios de la transición hacia una economía verde". En Uría, A. (coord.): De la economía verde a las sociedades verdes (pp. 9-24). Madrid: Los libros de La Catarata.

Throsby, D. (1998). "Cultural capital", conference on International Association for Cultural Economics. Barcelona.

Trotiño, L. (2012). "Las Ciudades Patrimonio de la Humanidad de la Región Turística de Madrid: Niveles de medios de funcionalidad y adecuación turística del Patrimonio Cultural". Papers de Turisme, $\mathrm{n}^{\circ}$ 51. Pp. 109-132.

Troitiño, M. A. (2002). "Aranjuez: Patrimonio cultural, recuperación urbana y turismo". Anales de Geografía de la Universidad Complutense. Extraordinario/2002. Pp. 495-518.

UNESCO (2014). Indicadores UNESCO de Cultura para el desarrollo. Manual Metodológico. París, UNESCO.

United Nations world tourism organization UNWTO (2013). Sustainable Tourism for Development Guidebook. Madrid. 
Vanblarcom, B. L. y Kayahan, C. (2011). "Assessing the economic impact of a UNESCO World Heritage designation". Journal of Heritage Tourism, 6(2): 143-164.

Weaver, D. (2009). "Reflections on sustainable tourism and paradigm change". En Gössling, S., Hall, M. y Weaver, D. (eds.) Sustainable tourism futures. Perspectives on systems, restructuring and innovations (pp. 33.40). New York: Routledge.

Yale, P. (1991). From tourist attractions to heritage tourism. Huntingdon: ELM publications

Zukin, S. (1995). The cultures of cities (Vol. 150). Oxford: Blackwell.

\section{Notas}

1 Investigación enmarcada en el proyecto “Guía Ciudades Legado Patrimonio Mundial en Madrid” (Ref. 346/2014), (2014-15) por encargo de la Subdirección General de Desarrollo y Sostenibilidad de la Dirección General de Turismo de la Comunidad de Madrid, subvencionado por la Asociación Plataforma Turística de Madrid. IP del Proyecto: Alicia Castillo Mena

2 https://www.turismomadrid.es/es/profesionales/estadisticas.html

3 https://www.madrid-destino.com/turismo/estadisticas

4 Encuesta de ocupación hotelera 2020, INE.

5 Desde el último cuarto del pasado siglo se ha desarrollado un sólido cuerpo científico que ha evidenciado las capacidades socioeconómicas del Patrimonio Cultural (Palma y Aguado 2010, Aguado et al 2017). Casos concretos: Bedate et al, 2004; Alonso \& Martín, 2008; Devesa et al, 2012; European Commission, 2012; Rizzo \& Mingosa, 2013; Barrio \& Herrero, 2014; Landriani \& Pozzoli, 2014; Herrero y Gómez, 2017.

6 Encuesta representativa según cuotas de sexo, grupo de edad y barrio de residencia. Intervalo de confianza: 95\%; margen de error: $7 \%$. Campo: junio y julio de 2015 .

7 Se seleccionaron al azar no residentes en una muestra equilibrada por género. Dado que no hay bases de datos que definan los grupos turísticos de estas ciudades, la muestra no pudo ser afijada por cuotas, por lo que las características de la población encuestada deben entenderse como indicativas del perfil y actitudes de la muestra.

8 Visitante sin pernoctación. Todo visitante que no pernocta en un medio de alojamiento colectivo o privado en el lugar visitado. Definición: Glosario Instituto de Estudios Turísticos. 